\title{
RESONANCE AND THE SECOND BVP
}

\author{
VICTOR L. SHAPIRO
}

\begin{abstract}
Let $\Omega \subset \mathbb{R}^{N}$ be a bounded open connected set with the cone property, and let $1<p<\infty$. Also, let $Q u$ be the $2 m$ th order quasilinear differential operator in generalized divergence form:

$$
Q u=\sum_{1 \leq|\alpha| \leq m}(-1)^{|\alpha|} D^{\alpha} A_{\alpha}\left(x, \xi_{m}(u)\right),
$$

where for $u \in W^{m, p}, \xi_{m}(u)=\left\{D^{\alpha} u:|\alpha| \leq m\right\}$. (For $m=1, Q u=$ $-\sum_{i=1}^{N} A_{i}(x, u, D u)$.) Under four assumptions on $A_{\alpha}$-Carathéodory, growth, monotonicity for $|\alpha|=m$, and ellipticity-results at resonance are established for the equation $Q u=G+f(x, u)$, where $G \in\left[W^{m, p}(\Omega)\right]^{*}$ and $f(x, u)$ satisfies a one-sided condition (plus others). For the case $m=1$, these results are tantamount to generalized solutions of the second BVP.
\end{abstract}

\section{INTRODUCTION}

Let $\Omega \subset \mathbb{R}^{N}, N \geq 1$, be a bounded open connected set with the cone property, i.e., there exists a finite cone $C$ such that each point $x$ in $\Omega$ is a vertex of a finite cone $C_{x}$ contained in $\Omega$ and congruent to $C$ (see $[2$, p. 11 or 1, p. 66]). The points of the open set $\Omega$ will be designated by $x=\left(x_{1}, \ldots, x_{N}\right)$, and the elementary differential operators by $D^{\alpha}=\prod_{j=1}^{N}\left(\partial / \partial x_{j}\right)^{\alpha_{j}}$ for an ordered $N$ tuple $\alpha=\left(\alpha_{1}, \ldots, \alpha_{N}\right)$ of nonnegative integers with the order of the operator $D^{\alpha}$ being written as $|\alpha|=\sum_{j=1}^{N} \alpha_{j}$. To write nonlinear partial differential operators in a convenient form, we introduce the vector space $\mathbb{R}^{s_{m}}$ whose elements are $\xi_{m}=\left\{\xi_{\alpha}:|\alpha| \leq m\right\}$, and divide each $\xi_{m}$ into two parts $\xi_{m}=\left(\eta_{m-1}, \zeta_{m}\right)$, where $\eta_{m-1}=\left\{\eta_{\beta}:|\beta| \leq m-1\right\} \in \mathbb{R}^{s_{m-1}}$ is the lower order part of $\xi_{m}$ and $\zeta_{m}=\left\{\zeta_{\alpha}:|\alpha|=m\right\}$ is the part of $\xi_{m}$ corresponding to the $m$ th derivatives. For $u \in W^{m, p}(\Omega), \xi_{m}(u)(x)=\left\{D^{\alpha} u(x):|\alpha| \leq m\right\}$. (Note $D^{(0,0, \ldots, 0)} u=u$.) In this paper, we shall study the $2 m$ th order differential operator in generalized divergence form:

$$
Q u=\sum_{1 \leq|\alpha| \leq m}(-1)^{|\alpha|} D^{\alpha} A_{\alpha}\left(x, \xi_{m}(u)\right)
$$

Received by the editors September 25, 1988 and, in revised form, May 2, 1989. Presented in part at the AMS meeting in Providence, R.I., August 8, 1988.

1980 Mathematics Subject Classification (1985 Revision). Primary 35J65; Secondary 35D05.

Key words and phrases. Quasilinear elliptic, resonance, generalized divergence form. 
For the functions $A_{\alpha}\left(x, \xi_{m}\right)$, we shall suppose

(A-1) Each $A_{\alpha}: \Omega \times \mathbb{R}^{s_{m}} \rightarrow \mathbb{R}$ satisfies the Carathéodory conditions (i.e., $A_{\alpha}\left(x, \xi_{m}\right)$ is measurable for $x$ in $\Omega$ for every fixed $\xi_{m} \in \mathbb{R}^{s_{m}}$ and continuous in $\xi_{m}$ for a.e. fixed $x \in \Omega$ ).

(A-2) $\exists$ constants $p$ and $c, 1<p<\infty, c \geq 0$, and a nonnegative function $\tilde{h} \in L^{p^{\prime}}(\Omega) \quad\left(p^{\prime}=p /(p-1)\right)$ such that

$$
\left|A_{\alpha}\left(x, \xi_{m}\right)\right| \leq \tilde{h}(x)+c\left|\xi_{m}\right|^{p-1}, \quad 1 \leq|\alpha| \leq m,
$$

for a.e. $x \in \Omega$ and for all $\xi_{m} \in \mathbb{R}^{s_{m}}$.

(A-3) $\sum_{|\alpha|=m}\left[A_{\alpha}\left(x, \eta_{m-1}, \zeta_{m}\right)-A_{\alpha}\left(x, \eta_{m-1}, \zeta_{m}^{\prime}\right)\right]\left(\zeta_{\alpha}-\zeta_{\alpha}^{\prime}\right)>0$ for a.e. $x \in$ $\Omega$ and for all $\left(\eta_{m-1}, \zeta_{m}\right) \in \mathbb{R}^{s_{m}}$ with $\zeta_{m} \neq \zeta_{m}^{\prime}$, where $A_{\alpha}\left(x, \xi_{m}\right)=$ $A_{\alpha}\left(x, \eta_{m-1}, \zeta_{m}\right)$ with $\xi_{m}=\left(\eta_{m-1}, \zeta_{m}\right)$.

(A-4) There exists a positive constant $c_{0}$ and a function $Z \in L^{1}(\Omega)$ such that

$$
\sum_{1 \leq|\alpha| \leq m} A_{\alpha}\left(x, \xi_{m}\right) \xi_{\alpha} \geq c_{0}\left\{\sum_{1 \leq|\alpha| \leq m}\left|\xi_{\alpha}\right|^{2}\right\}^{p / 2}-Z(x)
$$

for a.e. $x \in \Omega$ and for all $\xi_{m} \in \mathbb{R}^{s_{m}}$, where $p$ is the same constant as in (A-2).

Next, we introduce the semilinear form

$$
\mathscr{Q}(u, v)=\sum_{1 \leq|\alpha| \leq m} \int_{\Omega} A_{\alpha}\left(x, \xi_{m}(u)\right) D^{\alpha} v
$$

which because of (A-1) and (A-2) is well defined on $W^{m, p} \times W^{m, p}$. (For ease of notation, we henceforth designate $W^{m, p}(\Omega)$ by $W^{m, p}$.)

Theorem 1 which we will present shortly will also deal with a function $f(x, t): \Omega \times \mathbb{R} \rightarrow \mathbb{R}$ of the following nature:

(f-1) $f(x, t)$ meets the usual Caratheodory conditions.

(f-2) $\exists K^{\prime}>0$ and $\exists h_{1} \in L^{q^{\prime}}(\Omega)$ s.t. $|f(x, t)| \leq h_{1}(x)+K^{\prime}|t|^{q-1}$ for a.e. $x \in \Omega$ and for all $t \in \mathbb{R}$, where $q=p N /(N-m p)$ for $p<N m^{-1}$ and $q>p$ for $p \geq N m^{-1}$, and where $q^{\prime}=q /(q-1)$.

Also $h_{1}(x) \geq 0$ for a.e. $x \in \Omega$.

(f-3) $\exists h \in L^{q^{\prime}}(\Omega)$ s.t. $f(x, t) t \leq h(x)|t|$ for a.e. $x \in \Omega$ and for all $t \in \mathbb{R}$, where $h(x) \geq 0$ for a.e. $x \in \Omega$.

In the sequel $\left[W^{m, p}\right]^{*}$ will designate the dual space of $W^{m, p}$, i.e., the space of real bounded linear functionals defined on $W^{m, p}$.

We intend to prove two existence theorems at resonance for the differential operator $Q$ introduced in (1.1). These theorems will deal with generalized solutions of $Q$ given in terms of the semilinear form $\mathscr{Q}(u, v)$ introduced in (1.2). The first theorem we prove is the following. 
Theorem 1. Let $1<p<\infty$ and let $\Omega$ be a bounded open connected set with the cone property. Suppose $\mathscr{Q}(u, v)$ is given by (1.2) where $A_{\alpha}\left(x, \xi_{m}\right)$ satisfies (A-1)-(A-4) for $1 \leq|\alpha| \leq m$ and $f(x, t)$ satisfies (f-1)-(f-3). Also suppose that $G \in\left[W^{m, p}\right]^{*}$. Set $f_{+}(x)=\lim \sup _{t \rightarrow \infty} f(x, t)$ and $f_{-}(x)=$ $\liminf _{t \rightarrow-\infty} f(x, t)$ and suppose furthermore that

$$
\int_{\Omega} f_{+}(x)<-G(1)<\int_{\Omega} f_{-}(x) .
$$

Then $\exists u^{\#} \in W^{m, p}$ such that

$$
\mathscr{Q}\left(u^{\#}, v\right)=G(v)+\int_{\Omega} f\left(x, u^{\#}\right) v \quad \forall v \in W^{m, p} .
$$

Theorem 2 is a corollary to Theorem 1 and is presented at the end of this section.

From (1.2) and (A-4), we see that

$$
\mathscr{Q}(u, u) \geq c_{0} \int_{\Omega}\left\{\sum_{1 \leq|\alpha| \leq m}\left|D^{\alpha} u\right|^{2}\right\}^{p / 2}-\int_{\Omega} Z(x)
$$

for $u \in W^{m, p}$. Hence, we see that

$$
\liminf _{\|u\|_{L^{p}} \rightarrow \infty} \mathscr{Q}(u, u) /\|u\|_{L^{p}}^{p} \geq 0, \quad u \in W^{m, p} .
$$

Also, we see that if $u=$ constant, then $\mathscr{Q}(u, u)=0$. Therefore, if we compare (1.6) and this last fact with the situation that would arise if $Q$ were a linear operator (see $[6$, p. $213 ; 4$, p. 3]), we are well motivated to call Theorem 1 a theorem at resonance with the first eigenvalue $\lambda_{1}=0$ (i.e., if $G=0,(1.4)$ gives a generalized solution of the problem $\left.Q u=\lambda_{1} u+f(x, u)\right)$. Also we note that if $u \neq$ constant then

$$
\liminf _{t \rightarrow \infty} \mathscr{Q}(t u, t u) /\|t u\|_{L^{p}}^{p}>0 .
$$

Hence $\lambda_{1}=0$ acts as a simple eigenvalue.

Theorem 1 is motivated by the resonance theorems in $[8$, p. 611; 4, p. 13]. Condition (1.3) is usually referred to as a Landesman-Lazer condition. The connection between the above theorem and generalized solutions of the 2nd BVP (= Neumann BVP) for $Q$ in (1.1) becomes apparent if we temporarily restrict ourselves to the case $p=2, m=1$. It is well known in this situation that if the $\Omega$ we are dealing with also has the $C^{1}$-regularity property (see [1, pp. 67 and 114]), then $u \in W^{1,2}(\Omega)$ has a trace on the boundary of $\Omega$, i.e.,

$$
\exists K>0 \text { s.t. } \int_{\partial \Omega} u^{2} d \sigma \leq K\|u\|_{W^{1,2}}^{2} \quad \forall u \in W^{1,2} .
$$

Here, $\sigma$ represents the natural Borel measure on the boundary of our $C^{1}$ regular domain $\Omega$. In the sequel, when we say $g \in L^{2}(\partial \Omega)$, we shall mean $\int_{\partial \Omega} g^{2} d \sigma<\infty$. 
Since we are dealing with the $m=1$ case, $\xi_{1}=(\eta, \zeta)$, where $\eta \in \mathbb{R}$ and $\zeta=\left(\zeta_{1}, \ldots, \zeta_{N}\right) \in \mathbb{R}^{N}$. Hence we can write $Q$ in (1.1) as $Q u=$ $\sum_{i=1}^{N}-D_{i} A_{i}(x, u, D u)$, where $A_{i}\left(x, \xi_{1}\right)=A_{i}(x, \eta, \zeta), D_{i}=\partial / \partial x_{i}$, and $D=\left(D_{1}, \ldots, D_{N}\right)$. If $A_{i}(x, \eta, \zeta)=\sum_{j=1}^{N} a^{i j}(x) \zeta_{j}$ and

$$
Q u=\sum_{i=1}^{N}-D_{i}\left(\sum_{j=1}^{N} a^{i j}(x) D_{j} u\right)
$$

is strictly elliptic in $\Omega$ (see [6, p. 178]), and if furthermore $g \in L^{2}(\partial \Omega)$ and $f(x, t)$ meets (f-1), (f-2), and (f-3), then the 2nd BVP for $Q$ in $\Omega$ becomes

$$
\begin{gathered}
Q u=f(x, u) \quad \text { a.e. in } \Omega, \\
\sum_{i=1}^{N}\left(\sum_{j=1}^{N} a^{i j} D_{j} u\right) \nu_{i}=g \quad \text { a.e. on } \partial \Omega,
\end{gathered}
$$

where $\nu=\left(\nu_{1}, \ldots, \nu_{n}\right)$ is the outward pointing unit-normal for $\partial \Omega$. We say $u^{\#} \in W^{1,2}$ is a generalized solution of the 2nd BVP (i.e., of (1.8)) if the following prevails:

$$
\mathscr{Q}\left(u^{\#}, v\right)=\int_{\Omega} f\left(x, u^{\#}\right) v+\int_{\partial \Omega} g v d \sigma \quad \forall v \in W^{1,2},
$$

where $\mathscr{Q}\left(u^{\#}, v\right)$ is given by $(1.2)$, i.e., $\mathscr{Q}\left(u^{\#}, v\right)=\int_{\Omega}\left(\sum_{i, j=1}^{N} a^{i j}(x) D_{j} u^{\#} D_{i} v\right)$. This definition corresponds to the usual one for generalized solutions of the 2nd boundary value problem (see $[6$, p. $215 ; 7$, p. 160]).

Using (1.7), we see from Schwarz's inequality that $\int_{\partial \Omega} g v d \sigma=G(v)$, where $G \in\left[W^{1,2}\right]^{*}$. Therefore (1.9) becomes

$$
\mathscr{Q}\left(u^{\#}, v\right)=\int_{\Omega} f\left(x, u^{\#}\right) v+G(v) \quad \forall v \in W^{1,2}
$$

which is the same as (1.4). This gives the connection between Theorem 1 for the $p=2, m=1$ case and generalized solutions of the 2nd BVP.

For the case of general $p, 1<p<\infty$, and $m=1$, consider for example

$$
A_{i}(x, \eta, \zeta)=\sum_{j=1}^{N} a^{i j}(x) \zeta_{j}|\zeta|^{p-2}, \quad i=1, \ldots, N,
$$

where $a^{i j}(x)=0$ for $i \neq j$ and $a^{i i}(x)=a(x) \geq \varepsilon_{0}>0$ for $i=1, \ldots, N$ (i.e., $A_{1}(x, \eta, \xi)=a(x) \zeta_{1}|\zeta|^{p-2}$ for $\zeta \neq 0,=0$ for $\left.\zeta=0\right)$. It is easy to see that $A_{i}$ so defined meets conditions (A-1)-(A-4). If $g=0$ in (1.8), the second condition in (1.8) becomes

$$
\sum_{i=1}^{N} D_{i} u \nu_{i}=0 \text { a.e. on } \partial \Omega
$$

and (1.8) in this case can be viewed as the 2nd BVP with homogeneous boundary conditions (or the Neumann problem with homogeneous boundary conditions). 
$g=0$ gives rise to the case $G=0$. Hence, for $\Omega$ a bounded open set with the cone property, Theorem 1 with $G=0$ can be viewed as giving a generalized solution to the 2nd BVP with homogeneous boundary conditions for the case $m=1$ and $1<p<\infty$. Generalizing from this case, we see that in a certain sense we can view Theorem 1 as giving generalized solutions to the 2nd BVP for a bounded open set with the cone property all at resonance for our differential operator of order $2 m$ and $1<p<\infty$.

In order to state Theorem 2 , we need a different hypothesis than ( $f-3)$ given above, namely the following:

(f-4) Given $\varepsilon>0, \exists$ a nonnegative function $h_{\varepsilon}^{*} \in L^{q^{\prime}}(\Omega)$ and a constant $t_{0}(\varepsilon)$ s.t.

$$
t f(x, t) \leq \varepsilon|t|^{p}+h_{\varepsilon}^{*}(x)|t| \text { for } 1<p<\infty
$$

for $|t| \geq t_{0}(\varepsilon)$ and a.e. $x \in \Omega$.

We note that $(\mathrm{f}-4)$ is a generalization of the notion

$$
\limsup _{|t| \rightarrow \infty} f(x, t) / \operatorname{sgn} t|t|^{p-1} \leq 0
$$

uniformly for $x \in \Omega$. Motivated by [4, p. 1], we set

$$
\mathscr{F}_{ \pm}(x)=\limsup _{t \rightarrow \pm \infty} f(x, t) / \operatorname{sgn} t|t|^{p-1},
$$

and note that if $f(x, t)$ meets $(\mathrm{f}-4)$ above, then $\mathscr{F}_{ \pm}(x) \leq 0$ a.e. in $\Omega$.

We intend to establish the following theorem.

Theorem 2. Let $1<p<\infty$ and let $\Omega$ be a bounded open connected set with the cone property. Suppose that $\mathscr{Q}(u, v)$ is given by (1.2) where $A_{\alpha}\left(x, \xi_{m}\right)$ satisfies (A-1)-(A-4) and $f(x, t)$ satisfies (f-1), (f-2), and (f-4). Also suppose that $G \in\left[W^{m, p}\right]^{*}$. Let $\mathscr{F}_{ \pm}(x)$ be defined by (1.10), and suppose furthermore that

$$
\int_{\Omega} \mathscr{F}_{+}(x)<0 \text { and } \int_{\Omega} \mathscr{F}_{-}(x)<0 .
$$

Then $\exists u^{\#} \in W^{m, p}$ such that

$$
\mathscr{Q}\left(u^{\#}, v\right)=G(v)+\int_{\Omega} f\left(x, u^{\#}\right) v \quad \forall v \in W^{m, p} .
$$

Theorem 2 is essentially a corollary of Theorem 1 and will be established in $\S 4$ of this paper. The $\xi_{m}=\left(\eta_{m-1}, \zeta_{m}\right)$ notation introduced at the beginning of this section comes from Browder [3, p. 1]. Also, the author would like to acknowledge conversations with Professor James Stafney on the subject matter of this paper. Furthermore, the author would like to note that the proof of Proposition 1 given below has incorporated the use of property $\left(S_{+}\right)$at the suggestion of the referee. 


\section{A FUNDAMENTAL PROPOSITION}

Throughout this paper, we use the familiar notation

$$
\|u\|_{W^{m, p}}=\left\{\sum_{|\alpha| \leq m}\left\|D^{\alpha} u\right\|_{L^{p}}^{p}\right\}^{1 / p}
$$

for the norm in $W^{m, p}$, where $\|v\|_{L^{p}}=\left\{\int_{\Omega}|v|^{p}\right\}^{1 / p}$. For our fundamental proposition, we set

$$
f^{n}(x, t)= \begin{cases}f(x, n) & \text { if } t \geq n, \\ f(x, t) & \text { if }-n \leq t \leq n, \\ f(x,-n) & \text { if } t \leq-n .\end{cases}
$$

Also, we observe that for $q$ as in (f-2), it follows from [1, p. 97] that there exists a constant $K_{1}$ such that

$$
\|v\|_{L^{q}} \leq K_{1}\|v\|_{W^{m, p}} \quad \forall v \in W^{m, p} .
$$

We next prove the following proposition.

Proposition 1. Let $n$ be a positive integer and $\Omega$ be a bounded open connected set with the cone property. Suppose (i) $1<p<\infty$; (ii) $\mathscr{Q}(u, v)$ is given by (1.2) where $A_{\alpha}\left(x, \xi_{m}\right)$ satisfies (A-1)-(A-4) for $1 \leq|\alpha| \leq m$; (iii) $f(x, t)$ satisfies (f-1)-(f-3); and (iv) $G \in\left[W^{m, p}\right]^{*}$. Then $\exists u_{n} \in W^{m, p}$ which solves the following equation:

$$
\mathscr{Q}(u, v)+n^{-1} \int_{\Omega} \operatorname{sgn} u|u|^{p-1} v-\int_{\Omega} f^{n}(x, u) v=G(v) \quad \forall v \in W^{m, p} .
$$

To establish the above proposition we think of $n$ as a fixed positive integer and observe from $(\mathrm{f}-2)$ that

$$
\left|f^{n}(x, u)\right| \leq h_{1}(x)+K^{\prime} n^{q-1} \quad \forall u \in W^{m, p},
$$

where $h_{1} \in L^{q^{\prime}}$. Hence, we see from Hölder's inequality, (A-2), (1.2), (2.3), and (2.5) that for $u \in W^{m, p}$ there exists a constant $K(u)$ such that the absolute value of the left-hand side of (2.4) is majorized by $K(u)\|v\|_{W^{m, p}}$. Also the left-hand side of (2.4) is linear in $v$. Consequently the left-hand side of (2.4) defines implicitly an operator $T$ with the following two properties:

$$
T: W^{m, p} \rightarrow\left[W^{m, p}\right]^{*},
$$

where

$$
(T(u), v)=\mathscr{Q}(u, v)+n^{-1} \int_{\Omega} \operatorname{sgn} u|u|^{p-1} v-\int_{\Omega} f^{n}(x, u) v
$$

$\forall v \in W^{m, p}$. It follows also from Hölder's inequality, (A-2), (1.2), (2.3), and (2.5) that $T$ maps bounded sets in $W^{m, p}$ onto bounded sets in $\left[W^{m, p}\right]^{*}$. Therefore,

$T$ is a bounded operator. 
Next, we observe from (2.3) and (2.5) that there exists a constant $K_{2}$ such that

$$
\int_{\Omega}\left|f^{n}(x, u)\right||u| \leq K_{2}\|u\|_{W^{m, p}} \quad \forall u \in W^{m, p} .
$$

Also, we see from $(\mathrm{A}-4)$ that

$$
\mathscr{Q}(u, u) \geq c_{0} \int_{\Omega}\left\{\sum_{1 \leq|\alpha| \leq m}\left|D^{\alpha} u\right|^{2}\right\}^{p / 2}-\int_{\Omega} Z(x) .
$$

Consequently, since $n$ is a fixed positive integer, there exists a constant $K_{3}>0$ such that

$$
\mathscr{Q}(u, u)+n^{-1} \int_{\Omega}|u|^{p} \geq K_{3}\|u\|_{W^{m, p}}^{p}-\int_{\Omega} Z(x) \quad \forall u \in W^{m, p} .
$$

Since $1<p<\infty$, we conclude from (2.7), (2.9), and (2.10) that

$$
\lim _{\|u\|_{W^{m, p} \rightarrow \infty}} \frac{(T(u), u)}{\|u\|_{W^{m, p}}}=\infty
$$

We record this fact as

$$
T \text { is a coercive operator. }
$$

Next we show that

$$
T \text { is demicontinuous, }
$$

i.e., $u_{k} \rightarrow u$ in $W^{m, p}$ implies $T\left(u_{k}\right) \rightarrow T(u)$ in $\left[W^{m, p}\right]^{*}$. To establish (2.12), let $\left\{u_{k}\right\}_{k=1}^{\infty}$ be a sequence in $W^{m, p}$ and suppose

$$
\left\|u_{k}-u\right\|_{W^{m, p}} \rightarrow 0 \text { as } k \rightarrow \infty \text {. }
$$

Since $W^{m, p}[1$, p. 47] is a separable reflexive Banach space, (2.12) will follow if we can show there exists a subsequence $\left\{u_{k_{j}}\right\}_{j=1}^{\infty}$ such that

$$
\lim _{j \rightarrow \infty}\left(T\left(u_{k_{j}}\right), v\right)=(T(u), v) \quad \forall v \in W^{m, p} .
$$

To show that (2.14) holds, we observe from (2.13) that there exists a subsequence $\left\{u_{k_{j}}\right\}_{j=1}^{\infty}$ such that

$$
\lim _{j \rightarrow \infty} \xi_{m}\left(u_{k_{j}}(x)\right)=\xi_{m}(u(x)) \text { for a.e. } x \in \Omega \text {. }
$$

Next we observe from (2.13) and (A-2) that $\exists K_{4}>0$ such that

$$
\left\|A_{\alpha}\left(x, \xi_{m}\left(u_{k}\right)\right)\right\|_{L^{p^{\prime}}} \leq K_{4}
$$

for $1 \leq|\alpha| \leq m$ and $k=1,2, \ldots$. But then it follows from this last inequality that for fixed $v \in W^{m, p}$

$$
\left\{A_{\alpha}\left(x, \xi_{m}\left(u_{k}\right)\right) D^{\alpha} v\right\}_{k=1}^{\infty} \text { is absolutely equi-integrable }
$$


for $1 \leq|\alpha| \leq m$, i.e., given $\varepsilon>0, \exists \delta>0$ s.t. meas $E<\delta$ implies $\int_{E}\left|A_{\alpha}\left(x, u_{k}\right) D^{\alpha} v\right|<\varepsilon$ for $k=1,2, \ldots$ and $1 \leq|\alpha| \leq m$. To see this fact we apply Hölder's inequality and (2.16) to obtain

$$
\int_{E}\left|A_{\alpha}\left(x, \xi_{m}\left(u_{k}\right)\right) D^{\alpha} v\right| \leq K_{4}\left\{\int_{E} \mid\left(\left.D^{\alpha} v\right|^{p}\right\}^{1 / p} .\right.
$$

Since $v \in W^{m, p},(2.17)$ follows. From (2.15) and (A-1), we also see that

$$
\lim _{j \rightarrow \infty}\left[A_{\alpha}\left(x, \xi_{m}\left(u_{k_{j}}\right)\right)-A_{\alpha}\left(x, \xi_{m}(u)\right)\right] D^{\alpha} v=0 \text { a.e. } x \in \Omega
$$

for $1 \leq|\alpha| \leq m$. Consequently, it follows from (2.17) and Egoroff's theorem $[11$, p. 75$]$ that

$$
\lim _{j \rightarrow \infty} \int_{\Omega} A_{\alpha}\left(x, \xi_{m}\left(u_{k_{j}}\right)\right) D^{\alpha} v=\int_{\Omega} A_{\alpha}\left(x, \xi_{m}(u)\right) D^{\alpha} v \quad \forall v \in W^{m, p}
$$

and $1 \leq|\alpha| \leq m$.

In a similar manner, it follows that

$$
\lim _{j \rightarrow \infty} \int_{\Omega} \operatorname{sgn} u_{k_{j}}\left|u_{k_{j}}\right|^{p-1} v \quad \forall v \in W^{m, p} .
$$

Likewise using (f-1), (2.3), (2.5), and (2.15), it is an easy matter to show

$$
\lim _{j \rightarrow \infty} \int_{\Omega} f^{n}\left(x, u_{k_{j}}\right) v=\int_{\Omega} f^{n}(x, u) v \quad \forall v \in W^{m, p} .
$$

From (1.2), (2.7), (2.18), (2.19), and (2.20), we conclude that (2.14) holds. Hence, it follows that $T$ is a demicontinuous operator and (2.12) is established.

Next we show that

$$
T \text { satisfies the condition }\left(S_{+}\right) \text {, }
$$

i.e., if $u_{k}-u$ in $W^{m, p}$ and $\left.\varlimsup(T)\left(u_{k}\right)-T(u), u_{k}-u\right) \leq 0$, then $u_{k} \rightarrow u$ in $W^{m, p}$. Once (2.21) is established, it follows from (2.8), (2.12), and Necas $[10,3.3 .17$, p. 50$]$ that $T$ is a pseudomonotone operator (see [10, Definition 3.3.9]). Also, $W^{m, p}$ is a separable reflexive Banach space [1, p. 47]. Hence, it will follow from the pseudomonotonicity of $T,(2.8)$, and (2.11) in conjunction with Necas $[10,3.3 .6,3.3 .10-11, \mathrm{pp} .48-49]$ and (2.7) that a solution in $W^{m, p}$ exists for equation (2.4). Therefore to complete the proof of Proposition 1, it only remains to show that $(2.21)$ is indeed true.

Accordingly, we assume that the sequence $\left\{u_{k}\right\}_{k=1}^{\infty}$ has the following two properties:

$$
\begin{aligned}
& \lim _{k \rightarrow \infty} u_{k}=u \quad \text { weakly in } W^{m, p} ; \\
& \varlimsup_{k \rightarrow \infty}\left(T\left(u_{k}\right)-T(u), u_{k}-u\right) \leq 0 .
\end{aligned}
$$

To establish (2.21), it is clearly sufficient to establish the following:

(2.24) there exists a subsequence $\left\{u_{k_{j}}\right\}_{j=1}^{\infty}$ such that $\lim _{j \rightarrow \infty}\left\|u_{k_{j}}-u\right\|_{W^{m, p}}=0$. 
We now show that (2.22) and (2.23) together imply (2.24). To do this, we invoke the compact imbedding theorem for Sobolev spaces [1, p. 144] and apply this to the separable reflexive Banach space $W^{m, p}$ to obtain from (2.22) the following five statements for a subsequence (which for ease of notation we take to be the full sequence):

$$
\begin{gathered}
\exists K_{5}>0 \text { s.t. }\left\|u_{k}\right\|_{W^{m, p}} \leq K_{5} \quad \text { for } k=1,2, \ldots, \\
\lim _{k \rightarrow \infty}\left\|D^{\alpha} u_{k}-D^{\alpha} u\right\|_{L^{p}}=0 \quad \text { for }|\alpha| \leq m-1, \\
\lim _{k \rightarrow \infty} \int_{\Omega} D^{\alpha} u_{k} w=\int_{\Omega} D^{\alpha} u w \quad \forall w \in L^{p^{\prime}} \text { and }|\alpha|=m, \\
\lim _{k \rightarrow \infty}\left(T(u), u_{k}-u\right)=0, \\
\lim _{k \rightarrow \infty} \eta_{m-1}\left(u_{k}(x)\right)=\eta_{m-1}(u(x)) \text { for a.e. } x \in \Omega,
\end{gathered}
$$

where $\eta_{m-1}(u(x))=\left\{D^{\alpha} u(x):|\alpha| \leq m-1\right\}$.

Continuing with our attempt to establish (2.24), we next observe from (2.3) and (2.25) that there exists $K_{6}>0$ such that

$$
\int_{\Omega}\left|u_{k}-u\right|^{q} \leq K_{6}^{q} \quad \text { for } k=1,2, \ldots,
$$

and from (2.5) that for measurable $E \subset \Omega$

$$
\int_{E}\left|f^{n}\left(x, u_{k}\right)\right|\left|u_{k}-u\right| \leq K_{6}\left\{\int_{E}\left[h_{1}(x)+K^{\prime} n^{q-1}\right]^{q^{\prime}}\right\}^{1 / q^{\prime}} \quad \text { for } k=1,2, \ldots
$$

Consequently since $n$ is a fixed integer, it follows that $\left\{f^{n}\left(x, u_{k}\right)\left(u_{k}-u\right)\right\}_{k=1}^{\infty}$ is an absolutely equi-integrable sequence. From (f-1), (2.5), and (2.29), the terms of this last mentioned sequence converge to zero a.e. in $\Omega$. Hence, it follows from Egoroff's theorem [11, p. 75] that

$$
\lim _{k \rightarrow \infty} \int_{\Omega}\left[\left|f^{n}\left(x, u_{k}\right)\right|+\left|f^{n}(x, u)\right|\right]\left|u_{k}-u\right|=0 .
$$

Also, it follows from Hölder's inequality, (2.25), and (2.26) that

$$
\lim _{k \rightarrow \infty} \int_{\Omega}\left[\left|u_{k}\right|^{p-1}+|u|^{p-1}\right]\left|u_{k}-u\right|=0 .
$$

Consequently we conclude from (2.7), (2.23), (2.28), (2.31), and (2.32) that

$$
\varlimsup \mathscr{\operatorname { l i m }} \mathscr{Q}\left(u_{k}, u_{k}-u\right) \leq 0 \text {. }
$$

Using this fact along with (2.25)-(2.29), we next propose to show there exists a subsequence $\left\{u_{k_{j}}\right\}_{k=1}^{\infty}$ such that

$$
\lim _{k \rightarrow \infty} \zeta_{m}\left(u_{k_{j}}(x)\right)=\zeta_{m}(u(x)) \text { for a.e. } x \in \Omega,
$$


where $\zeta_{m}(u(x))=\left\{D^{\alpha} u(x):|\alpha|=m\right\}$. Once (2.34) is established it will be an easy matter to show that (2.24) holds. To establish (2.34), it is sufficient to establish the following two facts:

(1) There exists a subsequence $\left\{u_{k_{j}}\right\}_{j=1}^{\infty}$ such that

$$
\begin{aligned}
\lim _{j \rightarrow \infty} \sum_{|\alpha|=m} & {\left[A_{\alpha}\left(x, \eta_{m-1}\left(u_{k_{j}}\right), \zeta_{m}\left(u_{k_{j}}\right)\right)-A_{\alpha}\left(x, \eta_{m-1}\left(u_{k_{j}}\right), \zeta_{m}(u)\right)\right] } \\
\times & {\left[D^{\alpha} u_{k_{j}}(x)-D^{\alpha} u(x)\right]=0 \text { for a.e. } x \in \Omega, }
\end{aligned}
$$

where $\xi_{m}\left(u_{k_{j}}\right)=\left(\eta_{m-1}\left(u_{k_{j}}\right), \zeta_{m}\left(u_{k_{j}}\right)\right)$.

(2) With $\left\{u_{k_{j}}\right\}_{j=1}^{\infty}$ designating the same subsequence as in (2.35),

$$
\left\{\left|\zeta_{m}\left(u_{k_{j}}(x)\right)\right|\right\}_{j=1}^{\infty} \text { is pointwise bounded for a.e. } x \in \Omega,
$$

i.e., $\exists$ a finite constant $K(x)$ s.t.

$$
\left|\zeta_{m}\left(u_{k_{j}}(x)\right)\right| \leq K(x) \text { for } j=1,2, \ldots
$$

To see that (2.35) and (2.36) imply (2.34), let $\Omega_{1}$ be the subset of $\Omega$ for which (2.29), (2.35), and (2.36) all hold simultaneously for $\left\{u_{k_{j}}\right\}_{j=1}^{\infty}$. Consequently

$$
\text { meas } \Omega=\text { meas } \Omega_{1} \text {. }
$$

Suppose there exists $x_{0} \in \Omega_{1}$ for which the equality in (2.34) does not hold. Hence by (2.36) there exists a further subsequence $\left\{\zeta_{m}\left(u_{k_{j_{l}}}\left(x_{0}\right)\right)\right\}_{l=1}^{\infty}$ and a $\zeta_{m}^{*} \in \mathbb{R}^{s_{m}-s_{m-1}}$ with

$$
\zeta_{m}^{*} \neq \zeta_{m}\left(u\left(x_{0}\right)\right)
$$

such that $\lim _{l \rightarrow \infty} \zeta_{m}\left(u_{k_{j_{l}}}\left(x_{0}\right)\right)=\zeta_{m}^{*}$. Therefore from (2.29)

$$
\begin{aligned}
\lim _{l \rightarrow \infty} \sum_{|\alpha|=m}\left[A_{\alpha}\left(x_{0}, \eta_{m-1}\left(u_{k_{j_{l}}}\right), \zeta_{m}\left(u_{k_{j_{l}}}\right)\right)-A_{\alpha}\left(x_{0}, \eta_{m-1}\left(u_{k_{j_{l}}}\right), \zeta_{m}(u)\right)\right] \\
\times\left[D^{\alpha} u_{k_{j_{l}}}\left(x_{0}\right)-D^{\alpha}\left(u\left(x_{0}\right)\right)\right] \\
=\sum_{|\alpha|=m}\left[A_{\alpha}\left(x_{0}, \eta_{m-1}(u), \zeta_{m}^{*}\right)-A_{\alpha}\left(x_{0}, \eta_{m-1}(u), \zeta_{m}(u)\right)\right] \\
\times\left[\zeta_{m}^{*}-D^{\alpha} u\left(x_{0}\right)\right] .
\end{aligned}
$$

From (2.38) and (A-3) we see that the right-hand side of the equality in (2.39) is strictly positive. Hence the limit on the left-hand side of the equality in (2.39) is strictly positive. However $x_{0}$ is in $\Omega_{1}$ and from the choice of $\Omega_{1}$ and (2.35) we see that the limit on the left-hand side of the equality in (2.39) is zero. We have arrived at a contradiction. Consequently no such point like $x_{0}$ exists in $\Omega_{1}$. From (2.37), we have that the Lebesgue measure of $\Omega_{1}$ is the same as that of $\Omega$. We conclude that (2.34) does indeed hold once (2.35) and (2.36) are established. 
To establish (2.35), we shall show separately that

$$
\lim _{k \rightarrow \infty} \int_{\Omega} \sum_{|\alpha|=m} A_{\alpha}\left(x, \eta_{m-1}\left(u_{k}\right), \zeta_{m}(u)\right)\left[D^{\alpha} u_{k}(x)-D^{\alpha} u(x)\right]=0
$$

and

$$
\lim _{k \rightarrow \infty} \int_{\Omega} \sum_{|\alpha|=m} A_{\alpha}\left(x, \xi_{m}\left(u_{k}\right)\right)\left[D^{\alpha} u_{k}(x)-D^{\alpha} u(x)\right]=0 .
$$

To see that (2.35) follows from (2.40) and (2.41), we observe from the difference of these two limits that

$$
\begin{aligned}
& \lim _{k \rightarrow \infty} \int_{\Omega} \sum_{|\alpha|=m} {\left[A_{\alpha}\left(x, \eta_{m-1}\left(u_{k}\right), \zeta_{m}\left(u_{k}\right)\right)-A_{\alpha}\left(x, \eta_{m-1}\left(u_{k}\right), \zeta_{m}(u)\right)\right] } \\
& \times\left[D^{\alpha} u_{k}(x)-D^{\alpha} u(x)\right]=0 .
\end{aligned}
$$

But by (A-3), the integrand in this last limit is nonnegative for a.e. $x \in \Omega$. Hence the sequence

$$
\begin{aligned}
\left\{\sum _ { | \alpha | = m } \left[A_{\alpha}\left(x, \eta_{m-1}\left(u_{k}\right), \zeta_{m}\left(u_{k}\right)\right)-A_{\alpha}(x,\right.\right. & \left.\left.\eta_{m-1}\left(u_{k}\right), \zeta_{m}(u)\right)\right] \\
& \left.\times\left[D^{\alpha} u_{k}(x)-D^{\alpha} u(x)\right]\right\}_{k=1}^{\infty}
\end{aligned}
$$

converges in $L^{1}$-norm to zero, and (2.35) follows immediately from [11, p. 70].

To establish (2.40), we observe that

$$
\begin{aligned}
\int_{\Omega} A_{\alpha}\left(x, \eta_{m-1}\left(u_{k}\right), \zeta_{m}(u)\right)\left[D^{\alpha} u_{k}(x)-D^{\alpha} u(x)\right] \\
=\int_{\Omega}\left[A_{\alpha}\left(x, \eta_{m-1}\left(u_{k}\right), \zeta_{m}(u)\right)-A_{\alpha}\left(x, \eta_{m-1}(u), \zeta_{m}(u)\right)\right] \\
\quad \times\left[D^{\alpha} u_{k}-D^{\alpha} u\right] \\
\quad+\int_{\Omega} A_{\alpha}\left(x, \eta_{m-1}(u), \zeta_{m}(u)\right)\left[D^{\alpha} u_{k}(x)-D^{\alpha} u\right] .
\end{aligned}
$$

From $u \in W^{m, p}$ and (A-2), we see that $A_{\alpha}\left(x, \eta_{m-1}(u), \zeta_{m}(u)\right) \in L^{p^{\prime}}$ for $|\alpha|=m$. Consequently, it follows from (2.27) that the second integral on the right-hand side of the equality in (2.42) converges to zero as $k \rightarrow \infty$ for $|\alpha|=m$. Therefore (2.40) will follow once we show that

$$
\begin{aligned}
\lim _{k \rightarrow \infty} \int_{\Omega} & {\left[A_{\alpha}\left(x, \eta_{m-1}\left(u_{k}\right), \zeta_{m}(u)\right)-A_{\alpha}\left(x, \eta_{m-1}(u), \zeta_{m}(u)\right)\right] } \\
\times & {\left[D^{\alpha} u_{k}-D^{\alpha} u\right]=0 }
\end{aligned}
$$

for $|\alpha|=m$. From (2.25) and Hölder's inequality we see that this last limit will follow once we show

$$
\lim _{k \rightarrow \infty} \int_{\Omega}\left[A_{\alpha}\left(x, \eta_{m-1}\left(u_{k}\right), \zeta_{m}(u)\right)-A_{\alpha}\left(x, \eta_{m-1}(u), \zeta_{m}(u)\right)\right]^{p /(p-1)}=0
$$


for $|\alpha|=m$. To see that (2.43) holds, we observe from (2.29) and (A-1) that the integrand in (2.43) converges to zero as $k \rightarrow \infty$ for a.e. $x \in \Omega$. Also, we see from (2.26) and (A-2) that the integrand in (2.43) is absolutely equi-integrable, i.e., given $\varepsilon>0, \exists \delta$ s.t. meas $E<\delta \Rightarrow$

$$
\int_{E}\left|A_{\alpha}\left(x, \eta_{m-1}\left(u_{k}\right), \zeta_{m}(u)\right)-A_{\alpha}\left(x, \eta_{m-1}(u), \zeta_{m}(u)\right)\right|^{p /(p-1)}<\varepsilon
$$

for $|\alpha|=m$ and $k=1,2, \ldots$. Consequently, we conclude from Egoroff's theorem [11, p. 75], that $(2.43)$ holds. But this establishes (2.40). It remains to establish (2.41).

To establish (2.41), we observe from (A-2) and (2.25) that $\exists K_{7}>0$ such that

$$
\int_{\Omega}\left|A_{\alpha}\left(x, \xi_{m}\left(u_{k}\right)\right)\right|^{p^{\prime}} \leq K_{7}^{p^{\prime}} \quad \text { for }|\alpha|=m \text { and } k=1,2, \ldots .
$$

Consequently, it follows from (2.26) and Hölder's inequality that

$$
\lim _{k \rightarrow \infty} \int_{\Omega} A_{\alpha}\left(x, \xi_{m}\left(u_{k}\right)\right) D^{\alpha}\left(u_{k}-u\right)=0 \text { for } 0 \leq|\alpha| \leq m-1 .
$$

But then we obtain from (1.2) and (2.33) that

$$
\varlimsup_{k \rightarrow \infty} \int_{\Omega} \sum_{|\alpha|=m} A_{\alpha}\left(x, \xi_{m}\left(u_{k}\right)\right)\left[D^{\alpha} u_{k}(x)-D^{\alpha} u(x)\right] \leq 0 .
$$

Also, since $u \in W^{m, p}$, we have from (A-2) that $A_{\alpha}\left(x, \xi_{m}(u)\right) \in L^{p^{\prime}}$ for $|\alpha|=$ $m$. Therefore from (2.40), which we have already established, we obtain that

$$
\begin{gathered}
\varlimsup_{k \rightarrow \infty} \int_{\Omega} \sum_{|\alpha|=m}\left[A_{\alpha}\left(x, \xi_{m}\left(u_{k}\right)\right)-A_{\alpha}\left(x, \eta_{m-1}\left(u_{k}\right), \zeta_{m}(u)\right)\right] \\
\times\left[D^{\alpha} u_{k}(x)-D^{\alpha} u(x)\right] \leq 0 .
\end{gathered}
$$

But from (A-3) it follows that the integrand in this last expression is nonnegative for a.e. $x \in \Omega$ for $k=1,2, \ldots$. Consequently, the integral is nonnegative for every $k$, and we conclude that

$$
\begin{aligned}
& \lim _{k \rightarrow \infty} \int_{\Omega} \sum_{|\alpha|=m}[\left.A_{\alpha}\left(x, \xi_{m}\left(u_{k}\right)\right)-A_{\alpha}\left(x, \eta_{m-1}\left(u_{k}\right), \zeta_{m}(u)\right)\right] \\
& \times\left[D^{\alpha} u_{k}-D u\right]=0 .
\end{aligned}
$$

This last limit coupled with (2.40) gives (2.41). Since (2.40) has already been established, we have that $(2.35)$ is indeed true.

In order to establish (2.34), it remains to show that (2.36) holds. To accomplish this we proceed as follows.

Let $\Omega_{2} \subset \Omega$ be the set where the limits in (2.29) and (2.35) hold, where $\xi_{m}\left(u_{k_{j}}(x)\right), \xi_{m}(u(x)), A_{\alpha}\left(x, \eta_{m-1}\left(u_{k_{j}}(x)\right), \zeta_{m}(u(x))\right), \tilde{h}(x)$, and $Z(x)$ are finite-valued for $1 \leq|\alpha| \leq m$ and $j=1,2, \ldots$, and also where (A-2) and 
(A-4) hold. Then $\Omega_{2}$ has the same Lebesgue measure as $\Omega$, and to establish (2.36) it is sufficient to show that

$$
\left\{\left|\zeta_{m}\left(u_{k_{j}}(x)\right)\right|\right\}_{k=1}^{\infty} \text { is pointwise bounded for } x \in \Omega_{2} .
$$

To the contrary, suppose that there exist a point $x_{0} \in \Omega_{2}$ and a subsequence $\left\{\left|\zeta_{m}\left(u_{k_{j_{l}}}(x)\right)\right|\right\}_{l=1}^{\infty}$ such that

$$
\lim _{l \rightarrow \infty}\left|\zeta_{m}\left(u_{k_{j_{l}}}\left(x_{0}\right)\right)\right|=\infty
$$

Let $\varepsilon>0$ be such that $\varepsilon<1$ and $p-\varepsilon>1$. Then it follows from (A-4) that

$$
\begin{aligned}
& c_{0}\left|\zeta_{m}\left(u_{k_{j_{l}}}\left(x_{0}\right)\right)\right|^{p} \\
& \quad \leq \sum_{1 \leq|\alpha| \leq m} A_{\alpha}\left(x_{0}, \eta_{m-1}\left(u_{k_{j_{l}}}\right), \zeta_{m}\left(u_{k_{j}}\right)\right) D^{\alpha} u_{k_{j_{l}}}\left(x_{0}\right)+Z\left(x_{0}\right) .
\end{aligned}
$$

Also, for fixed $k$, we have that

$$
\begin{aligned}
A_{\alpha}(x, & \left.\xi_{m}\left(u_{k}(x)\right)\right) D^{\alpha} u_{k}(x) \\
= & A_{\alpha}\left(x, \xi_{m}\left(u_{k}(x)\right)\right) D^{\alpha} u(x) \\
& +A_{\alpha}\left(x, \eta_{m-1}\left(u_{k}\right), \zeta_{m}(u)\right)\left[D^{\alpha} u_{k}(x)-D^{\alpha} u(x)\right] \\
& +\left[A_{\alpha}\left(x, \xi_{m}\left(u_{k}\right)\right)-A_{\alpha}\left(x, \eta_{m-1}\left(u_{k}\right), \zeta_{m}(u)\right)\right] \\
& \times\left[D^{\alpha} u_{k}(x)-D^{\alpha} u(x)\right] .
\end{aligned}
$$

Next, we observe from (A-2), (2.29), (2.49), and the definition of $\Omega_{2}$ that both

$$
\begin{aligned}
& \lim _{l \rightarrow \infty} A_{\alpha}(\left.x_{0}, \xi_{m}\left(u_{k_{j_{l}}}\right)\right) D^{\alpha} u\left(x_{0}\right) /\left|\zeta_{m}\left(u_{k_{j_{l}}}\left(x_{0}\right)\right)\right|^{p-\varepsilon}=0, \\
& \lim _{l \rightarrow \infty} A_{\alpha}(\left.x_{0}, \eta_{m-1}\left(u_{k_{j_{l}}}\right), \zeta_{m}(u)\right) \\
& \quad \times\left[D^{\alpha} u_{k_{j_{l}}}\left(x_{0}\right)-D^{\alpha} u\left(x_{0}\right)\right] /\left|\zeta_{m}\left(u_{k_{j_{l}}}\left(x_{0}\right)\right)\right|^{p-\varepsilon}=0
\end{aligned}
$$

for $1 \leq|\alpha| \leq m$. Also from the same observation we have

$$
\begin{aligned}
\lim _{l \rightarrow \infty}\left[A_{\alpha}\left(x_{0}, \xi_{m}\left(u_{k_{j_{l}}}\right)\right)-A_{\alpha}\left(x_{0}, \eta_{m-1}\left(u_{k_{j_{l}}}\right), \zeta_{m}(u)\right)\right] & \\
\times & {\left[D^{\alpha} u_{k_{j_{l}}}\left(x_{0}\right)-D^{\alpha} u\left(x_{0}\right)\right] /\left|\zeta_{m}\left(u_{k_{j_{l}}}\left(x_{0}\right)\right)\right|^{p-\varepsilon}=0 }
\end{aligned}
$$

for $1 \leq|\alpha| \leq m-1$. Furthermore, from (2.35), (2.49) and the definition of $\Omega_{2}$ we have

$$
\begin{aligned}
\lim _{l \rightarrow \infty} \sum_{|\alpha|=m}\left\{\left[A_{\alpha}\left(x_{0}, \xi_{m}\left(u_{k_{j_{l}}}\right)\right)-A_{\alpha}\left(x_{0}, \eta_{m-1}\left(u_{k_{j_{l}}}\right), \zeta_{m}\left(u_{k_{j_{l}}}\right)\right)\right]\right. \\
\left.\times\left[D^{\alpha} u_{k_{j_{l}}}\left(x_{0}\right)-D^{\alpha} u\left(x_{0}\right)\right]\right\} /\left|\zeta_{m}\left(u_{k_{j_{l}}}\left(x_{0}\right)\right)\right|^{p-\varepsilon}=0 .
\end{aligned}
$$

Dividing both sides of (2.50) by $\left|\zeta_{m}\left(u_{k_{j_{l}}}\left(x_{0}\right)\right)\right|^{p-\varepsilon}$ and using (2.51)-(2.54), we obtain that

$$
c_{0} \lim _{l \rightarrow \infty}\left|\zeta_{m}\left(u_{k_{j_{l}}}\left(x_{0}\right)\right)\right|^{\varepsilon}=0
$$


Since $c_{0}$ is positive, this gives that

$$
\lim _{l \rightarrow \infty}\left|\zeta_{m}\left(u_{k_{j_{l}}}\left(x_{0}\right)\right)\right|^{\varepsilon}=0 .
$$

But this fact is a direct contradiction of (2.49). We conclude that (2.48) is true. Since $\Omega-\Omega_{2}$ is a set of Lebesgue measure zero, (2.36) is established. As we have shown earlier, (2.35) and (2.36) imply that (2.34) holds. Hence (2.34) is established.

It remains to show that $(2.24)$ holds. In order to do this we observe that

$$
\left\{\left|\zeta_{m}\left(u_{k_{j_{l}}}(x)\right)\right|^{p}\right\}_{j=1}^{\infty} \text { is absolutely equi-integrable. }
$$

This fact follows from (2.50) with $x_{0}$ replaced by $x,(2.51)$, and the following four observations:

$$
\left\{A_{\alpha}\left(x, \xi_{m}\left(u_{k_{j}}(x)\right)\right) D^{\alpha} u\right\}_{j=1}^{\infty} \text { is absolutely equi-integrable }
$$

for $1 \leq|\alpha| \leq m$;

$$
\left\{A_{\alpha}\left(x, \eta_{m-1}\left(u_{k_{j}}\right), \zeta_{m}(u)\right)\left[D^{\alpha} u_{k_{j}}-D^{\alpha} u\right]\right\}_{j=1}^{\infty} \quad \text { is absolutely equi-integrable }
$$

for $1 \leq|\alpha| \leq m$;

$$
\begin{gathered}
\left\{\left[A_{\alpha}\left(x, \xi_{m}\left(u_{k_{j}}\right)\right)-A_{\alpha}\left(x, \eta_{m-1}\left(u_{k_{j}}\right), \zeta_{m}(u)\right)\right]\left[D^{\alpha} u_{k_{j}}-D^{\alpha} u\right]\right\}_{j=1}^{\infty} \\
\text { is absolutely equi-integrable }
\end{gathered}
$$

for $1 \leq|\alpha| \leq m-1$;

$$
\text { the integrand in }(2.41)^{\prime} \text { is nonnegative. }
$$

Next, we observe from (2.34) that

$$
\lim _{j \rightarrow \infty}\left|D^{\alpha} u_{k_{j}}(x)-D^{\alpha} u(x)\right|^{p}=0 \text { a.e. in } \Omega
$$

for $|\alpha|=m$. From (2.55), we have that

$$
\left\{\left|D^{\alpha} u_{k_{j}}-D^{\alpha} u\right|^{p}\right\}_{j=1}^{\infty} \text { is absolutely equi-integrable for }|\alpha|=m .
$$

Hence, it follows from Egoroff's theorem that

$$
\lim _{j \rightarrow \infty} \int_{\Omega}\left|D^{\alpha} u_{k_{j}}-D^{\alpha} u\right|^{p}=0 \quad \text { for }|\alpha|=m .
$$

This fact coupled with (2.26) gives (2.24). Hence $T$ satisfies condition $\left(S_{+}\right)$, and the proof of the proposition is complete.

\section{Proof of Theorem 1}

To prove Theorem 1 we invoke Proposition 1 and obtain a sequence $\left\{u_{n}\right\}_{n=1}^{\infty}$ such that

$$
u_{n} \in W^{m, p} \quad \text { satisfies }(2.4) \text { for } n=1,2, \ldots
$$


We claim that

$$
\left\{\left\|u_{n}\right\|_{W^{m, p}}\right\}_{n=1}^{\infty} \text { is a uniformly bounded sequence. }
$$

Suppose the claim in (3.2) is false. Then without loss in generality, we can assume

$$
\lim _{n \rightarrow \infty}\left\|u_{n}\right\|_{W^{m, p}}=\infty \text {. }
$$

Replacing $v$ with $u_{n}$ in (2.4), we notice the first integral on the left-hand side is nonnegative, and apply (A-4) and (f-3) to obtain

$$
c \int_{\Omega}\left\{\sum_{1 \leq|\alpha| \leq m}\left|D^{\alpha} u_{n}\right|^{2}\right\}^{p / 2} \leq G\left(u_{n}\right)+\int_{\Omega} h(x)\left|u_{n}\right|+\int_{\Omega} Z(x) \text {, }
$$

where $h \in L^{q^{\prime}}$ and $c>0$. From (2.3), this last inequality, and the fact that $G \in\left[W^{m, p}\right]^{*}$, we see there is a constant $K_{8}$ such that

$$
\int_{\Omega}\left\{\sum_{1 \leq|\alpha| \leq m}\left|D^{\alpha} u_{n}\right|^{2}\right\}^{p / 2} \leq K_{8}\left\|u_{n}\right\|_{W^{m, p}}+\int_{\Omega} Z(x) .
$$

Setting

$$
v_{n}=u_{n} /\left\|u_{n}\right\|_{W^{m, p}}
$$

and dividing both sides of (3.4) by $\left\|u_{n}\right\|_{W^{m, p}}^{p}$, we obtain

$$
\int_{\Omega}\left\{\sum_{1 \leq|\alpha| \leq m}\left|D^{\alpha} v_{n}\right|^{2}\right\}^{p / 2} \leq K_{8}\left\|u_{n}\right\|_{W^{m, p}}^{1-p}+\int_{\Omega} Z(x)\left\|u_{n}\right\|_{W^{m, p}}^{-p} .
$$

Now $p>1$, and we conclude from (3.3) and this last inequality that

$$
\lim _{n \rightarrow \infty} \int_{\Omega}\left|D^{\alpha} v_{n}\right|^{p}=0 \quad \text { for } 1 \leq|\alpha| \leq m \text {. }
$$

Next, from (3.5) we see that

$$
\left\|v_{n}\right\|_{W^{m, p}}=1 \text { for } n=1,2, \ldots
$$

Hence, $\left\|v_{n}\right\|_{W^{m, p}}^{p}=1$, and from (2.1) we obtain that

$$
1=\left\|v_{n}\right\|_{L^{p}}^{p}+\sum_{1 \leq|\alpha| \leq m}\left\|D^{\alpha} v_{n}\right\|_{L^{p}}^{p} .
$$

We infer from (3.6) and this last equality that

$$
\lim _{n \rightarrow \infty}\left\|v_{n}\right\|_{L^{p}}=1 \text {. }
$$

Next, we see from (3.7) that $\left\{\left\|v_{n}\right\|_{W^{m, p}}\right\}_{n=1}^{\infty}$ is a uniformly bounded sequence. Consequently we obtain from the compact imbedding theorem [1, $\mathrm{p}$. 144] and the fact that $W^{m, p}$ is a separable reflexive Banach space [1, p. 47] 
the existence of a subsequence (which for ease of notation, we take to be the full sequence) and a function $v_{0}$ with the following properties:

$$
\begin{gathered}
v_{0} \in W^{m, p}, \\
\lim _{n \rightarrow \infty}\left\|D^{\alpha} v_{n}-D^{\alpha} v_{0}\right\|_{L^{p}}=0 \quad \text { for }|\alpha| \leq m-1, \\
\lim _{n \rightarrow \infty} \int_{\Omega} D^{\alpha} v_{n} w=\int_{\Omega} D^{\alpha} v_{0} w \quad \forall w \in L^{p^{\prime}} \text { and }|\alpha|=m, \\
\lim _{n \rightarrow \infty} G\left(v_{n}\right)=G\left(v_{0}\right), \\
\lim _{n \rightarrow \infty} D^{\alpha} v_{n}(x)=D^{\alpha} v_{0}(x) \quad \text { for a.e. } x \in \Omega \text { and }|\alpha| \leq m-1 .
\end{gathered}
$$

Also from (2.3),

$$
\left\{\left\|v_{n}\right\|_{L^{q}}\right\}_{n=1}^{\infty} \text { is a uniformly bounded sequence. }
$$

With $h$ as in (f-3), i.e., $h \in L^{q^{\prime}}$, it follows from (3.14) and Hölder's inequality that the sequence $\left\{h v_{n}\right\}_{n=1}^{\infty}$ is absolutely equi-integrable. Consequently, it follows from (3.13) and Egoroff's theorem that

$$
\lim _{n \rightarrow \infty} \int_{\Omega} h\left|v_{n}\right|=\int_{\Omega} h\left|v_{0}\right| \text {. }
$$

Next, we observe from (3.6), (3.10), and (3.11) that $\int_{\Omega} D^{\alpha} v_{0} w=0$ for $w \in L^{p^{\prime}}$ and $1 \leq|\alpha| \leq m$. Consequently, $D^{\alpha} v_{0}=0$ a.e! in $\Omega$ for $1 \leq|\alpha| \leq m$. Since $\Omega$ is a bounded open connected set, we conclude that $v_{0}=$ constant a.e. in $\Omega$. From (3.8) and (3.10), we obtain that $\left\|v_{0}\right\|_{L^{p}}=1$. Hence this constant is not zero. Therefore, this constant is either positive or negative. We shall assume it is positive. A similar argument will work in case it is negative. Hence, we assume

$$
v_{0}=c_{4} \text { for a.e. } x \in \Omega,
$$

where $c_{4}=$ [meas $\left.\Omega\right]^{-1 / p}$. Continuing with the proof, we invoke (3.1) and put $v_{n}=u_{n} /\left\|u_{n}\right\|_{W^{m, p}}$ in place of $v$ in (2.4) and observe from (1.2) and (A-4) that

$$
-\left\|u_{n}\right\|_{W^{m, p}}^{-1} \int_{\Omega} Z(x) \leq G\left(v_{n}\right)+\int_{\Omega} f^{n}\left(x, u_{n}\right) v_{n}
$$

for $n=1,2, \ldots$. Consequently, using (f-3), we infer from (3.17) that

$$
\int_{\Omega}\left[h(x)\left|v_{n}\right|-f^{n}\left(x, u_{n}\right) v_{n}\right]-\int_{\Omega} h(x)\left|v_{n}\right| \leq G\left(v_{n}\right)+\left\|u_{n}\right\|_{W^{m, p}}^{-1} \int_{\Omega} Z(x) .
$$

Since $Z \in L^{1}(\Omega)$, we obtain from (3.3), (3.12), and this last inequality that

$$
\liminf _{n \rightarrow \infty}\left\{\int_{\Omega}\left[h(x)\left|v_{n}\right|-f^{n}\left(x, u_{n}\right) v_{n}\right]-\int_{\Omega} h(x)\left|v_{n}\right|\right\} \leq G\left(v_{0}\right) .
$$


From (f-3) we have $h(x)\left|v_{n}\right|-f^{n}\left(x, u_{n}\right) v_{n} \geq 0$ a.e. in $\Omega$. Hence, using Fatou's lemma [11, p. 24] in conjunction with (3.13), (3.15), and (3.16), we obtain from (3.18) that

$$
-c_{4} \int_{\Omega} \limsup _{n \rightarrow \infty} f^{n}\left(x, u_{n}\right) \leq G\left(c_{4}\right) .
$$

Now $u_{n}=v_{n}\left\|u_{n}\right\|_{W^{m, p}}$. Therefore we have from (3.3), (3.13), and (3.16) that $\lim _{n \rightarrow \infty} u_{n}(x)=\infty$ a.e. in $\Omega$. It is an easy matter to check from (2.2) that lim sup $\operatorname{si\infty }_{n \rightarrow \infty} f^{n}\left(x, u_{n}\right) \leq f_{+}(x)$. (Note also from (f-3) that $f_{+}(x) \leq h(x)$ a.e. in $\Omega$.) Consequently, we conclude from (3.19) and the fact that $c_{4}$ is a positive constant that $-G(1) \leq \int_{\Omega} f_{+}(x)$. But this fact contradicts the first inequality in assumption (1.3). Hence (3.3) is false and claim (3.2) is established.

One notes from (3.2) there exists a constant $K_{9}$ such that

$$
\left\|u_{n}\right\|_{W^{m, p}} \leq K_{9} \text { for } n=1,2, \ldots .
$$

It therefore follows from the compact imbedding theorem [1, p. 144] and the fact that $W^{m, p}$ is a separable reflexive Banach space that there exists a subsequence (which for ease of notation we take to be the full sequence) and a function $u^{\#}$ such that

$$
\begin{gathered}
u^{\#} \in W^{m, p}, \\
\lim _{n \rightarrow \infty}\left\|D^{\alpha} u_{n}-D^{\alpha} u^{\#}\right\|_{L^{p}}=0 \quad \text { for }|\alpha| \leq m-1, \\
\lim _{n \rightarrow \infty} \int_{\Omega} D^{\alpha} u_{n} w=\int_{\Omega} D^{\alpha} u^{\#} w \quad \forall w \in L^{p^{\prime}} \text { and }|\alpha|=m, \\
\lim _{n \rightarrow \infty} G\left(u_{n}\right)=G\left(u^{\#}\right), \\
\lim _{n \rightarrow \infty} \eta_{m-1}\left(u_{n}(x)\right)=\eta_{m-1}\left(u^{\#}(x)\right) \text { for a.e. } x \in \Omega .
\end{gathered}
$$

We next propose to show there exists a subsequence $\left\{u_{n_{k}}\right\}_{k=1}^{\infty}$ s.t.

$$
\lim _{k \rightarrow \infty} \zeta_{m}\left(u_{n_{k}}(x)\right)=\zeta_{m}\left(u^{\#}(x)\right) \text { for a.e. } x \in \Omega \text {. }
$$

As in the proof of Proposition 1, once (3.26) is established, it will be an easy matter to establish Theorem 1 from (3.20)-(3.26).

To establish (3.26), it is sufficient to establish the following two facts:

(1) $\exists$ a subsequence $\left\{u_{n_{k}}\right\}_{k=1}^{\infty}$ s.t.

$$
\begin{aligned}
& \lim _{k \rightarrow \infty} \sum_{|\alpha|=m} {\left[A_{\alpha}\left(x, \eta_{m-1}\left(u_{n_{k}}\right), \zeta_{m}\left(u_{n_{k}}\right)\right)-A_{\alpha}\left(x, \eta_{m-1}\left(u_{n_{k}}\right), \zeta_{m}\left(u^{\#}\right)\right)\right] } \\
& \times\left[D^{\alpha} u_{n_{k}}(x)-D^{\alpha} u^{\#}(x)\right]=0 \text { for a.e. } x \in \Omega
\end{aligned}
$$

where $\xi_{m}\left(u_{n_{k}}\right)=\left(\eta_{m-1}\left(u_{n_{k}}\right), \zeta_{m}\left(u_{n_{k}}\right)\right)$. 
(2) With $\left\{u_{n_{k}}\right\}_{k=1}^{\infty}$ designating the same subsequence as in (3.27),

$$
\left\{\left|\zeta_{m}\left(u_{n_{k}}(x)\right)\right|\right\}_{k=1}^{\infty} \text { is pointwise bounded for a.e. } x \in \Omega \text {. }
$$

The proof that (3.27) and (3.28) imply (3.26) is the same that is used in the proof of Proposition 1 to show that (2.35) and (2.36) imply (2.34).

To establish (3.27) we show separately that

$$
\lim _{n \rightarrow \infty} \int_{\Omega} \sum_{|\alpha|=m} A_{\alpha}\left(x, \eta_{m-1}\left(u_{n}\right), \zeta_{m}\left(u^{\#}\right)\right)\left[D^{\alpha} u_{n}(x)-D^{\alpha} u^{\#}(x)\right]=0
$$

and

$$
\varlimsup_{n \rightarrow \infty} \int_{\Omega} \sum_{|\alpha|=m} A_{\alpha}\left(x, \xi_{m}\left(u_{n}\right)\right)\left[D^{\alpha} u_{n}(x)-D^{\alpha} u^{\#}(x)\right] \leq 0 .
$$

Once again the proof that (3.29) and (3.30) imply (3.27) is similar to that which was used in Proposition 1 to show that (2.40) and (2.41) imply (2.35) using (A-3) and [11, p. 70]. We leave the details to the reader. Also the proof that (3.29) holds is the same as the proof given for (2.40) in Proposition 1. We leave the details to the reader.

We now establish (3.30). We observe from (A-2) and (3.20) that there exists a $K_{10}$ such that

$$
\int_{\Omega}\left|A_{\alpha}\left(x, \xi_{m}\left(u_{n}\right)\right)\right|^{p /(p-1)} \leq K_{10}^{p^{\prime}} \text { for } 1 \leq|\alpha| \leq m \text { and } n=1,2, \ldots
$$

Consequently, we obtain from (3.22) and Hölder's inequality that

$$
\lim _{n \rightarrow \infty} \int_{\Omega} A_{\alpha}\left(x, \xi_{m}\left(u_{n}\right)\right)\left[D^{\alpha} u_{n}-D^{\alpha} u^{\#}\right]=0 \quad \text { for } 1 \leq|\alpha| \leq m-1 .
$$

Hence (3.30) will follow once we show

$$
\varlimsup_{n \rightarrow \infty} \int_{\Omega} \sum_{1 \leq|\alpha| \leq m} A_{\alpha}\left(x, \xi_{m}\left(u_{n}\right)\right)\left[D^{\alpha} u_{n}-D^{\alpha} u^{\#}\right] \leq 0 .
$$

Now from (1.2), we see that (3.32) is the same as

$$
\varlimsup_{n \rightarrow \infty} \mathscr{Q}\left(u_{n}, u_{n}-u^{\#}\right) \leq 0 \text {. }
$$

Hence, (3.30) will follow once we show that (3.33) holds. To establish (3.33), we use (3.1) and replace $v$ in (2.4) by $u_{n}-u^{\#}$ to obtain

$$
\begin{aligned}
\mathscr{Q}\left(u_{n}, u_{n}-u^{\#}\right)= & G\left(u_{n}-u^{\#}\right)+\int_{\Omega} f^{n}\left(x, u_{n}\right)\left(u_{n}-u^{\#}\right) \\
& -n^{-1} \int_{\Omega} \operatorname{sgn} u_{n}\left|u_{n}\right|^{p-1}\left(u_{n}-u^{\#}\right) .
\end{aligned}
$$

From (3.20), we see that $\left\{\left\|u_{n}\right\|_{L^{p}}\right\}$ is a uniformly bounded sequence. Also from (3.22) we have that $\lim _{n \rightarrow \infty}\left\|u_{n}-u^{\#}\right\|_{L^{p}}=0$. Hence it follows from Hölder's inequality that

$$
\lim _{n \rightarrow \infty} n^{-1} \int_{\Omega} \operatorname{sgn} u_{n}\left|u_{n}\right|^{p-1}\left(u_{n}-u^{\#}\right)=0
$$


We claim

$$
\varlimsup_{n \rightarrow \infty} \int_{\Omega} f^{n}\left(x, u_{n}\right)\left(u_{n}-u^{\#}\right) \leq 0 .
$$

To establish (3.36), we observe from (2.3) and (3.20) that there exists a constant $K_{11}$ such that

$$
\left\|u_{n}\right\|_{L^{q}} \leq K_{11} \text { for } n=1,2, \ldots \text {. }
$$

Next we observe from (f-1) and (3.25) that $\lim _{n \rightarrow \infty} f^{n}\left(x, u_{n}\right)=f\left(x, u^{\#}\right)$ a.e. in $\Omega$. Hence it follows from (3.25) that

$$
\lim _{n \rightarrow \infty} f^{n}\left(x, u_{n}\right)\left(u_{n}-u^{\#}\right)=0 \text { a.e. in } \Omega \text {. }
$$

Also given $\varepsilon>0$, we observe $\exists \delta>0$ such that if (meas $E$ ) $<\delta$ then

(i) $\int_{E}\left|f^{n}\left(x, u_{n}\right) u^{\#}\right|<\varepsilon$ for $n=1,2, \ldots$,

(ii) $\int_{E} f^{n}\left(x, u_{n}\right) u_{n}<\varepsilon$ for $n=1,2, \ldots$.

To see that (3.39)(i) holds, we observe from (f-2) that $\left|f^{n}\left(x, u_{n}\right)\right| \leq h_{1}(x)+$ $K^{\prime}\left|u_{n}\right|^{q-1}$ a.e. in $\Omega$ where $h_{1} \in L^{q^{\prime}}$ for $n=1,2, \ldots$. Hence it follows from (3.37) that $\left\{\int_{\Omega}\left|f^{n}\left(x, u_{n}\right)\right|^{q^{\prime}}\right\}_{n=1}^{\infty}$ is a uniformly bounded sequence, and (3.39)(i) follows immediately from Hölder's inequality and the fact that $u^{\#} \in$ $L^{q}$.

To establish (3.39)(ii), we observe from (f-3) and (3.37) that

$$
\int_{E} f^{n}\left(x, u_{n}\right) u_{n} \leq \int_{E} h u_{n} \leq\left\{\int_{E}|h|^{q^{\prime}}\right\}^{1 / q^{\prime}} K_{11} .
$$

Since $h \in L^{q^{\prime}},(3.39)$ (ii) follows from this last inequality.

To establish (3.36), let $\varepsilon>0$ be given and choose $\delta>0$ such that if (meas $E)<\delta,(3.39)(\mathrm{i})$ and (ii) hold. Next, using Egoroff's theorem in conjunction with (3.38), we see $\exists E$ with (meas $E$ ) $<\delta$ such that

$$
\lim _{n \rightarrow \infty} f^{n}\left(x, u_{n}\right)\left(u_{n}-u^{\#}\right)=0 \text { uniformly in } \Omega-E \text {. }
$$

Consequently, $\exists n_{0}$ such that for $n \geq n_{0}$

$$
\left|f^{n}\left(x, u_{n}\right)\left(u_{n}-u^{\#}\right)\right|<\varepsilon(\text { meas } \Omega)^{-1} \text { for } x \in \Omega-E .
$$

It therefore follows from (3.39)(i) and (ii) and this last fact that

$$
\int_{\Omega} f^{n}\left(x, u_{n}\right)\left(u_{n}-u^{\#}\right) \leq 3 \varepsilon \text { for } n \geq n_{0} .
$$

Since $\varepsilon$ is an arbitrary positive number, (3.36) follows immediately from this last inequality.

From (3.34), (3.24), (3.35), and (3.36), we see that (3.33) does indeed hold. Since (3.33) implies (3.30) via (3.32), we see also that (3.30) is established. Since (3.29) is already established, (3.27) follows from (A-3) and [11, p. 70]. It remains to establish (3.28). Now the proof that (3.28) holds is the same as 
the proof that (2.36) holds in the proof of Proposition 1. Replace $u_{k}$ by $u_{n}$, $u_{k_{j}}$ by $u_{n_{k}}, u_{k_{j_{j}}}$ by $u_{n_{k_{l}}}$, and $u$ by $u^{\#}$ and (3.28) follows. Hence (3.28) is established. As we have already observed, (3.27) and (3.28) imply (3.26).

To complete the proof of the theorem, we have to show that (3.20)-(3.26) along with (3.1) gives (1.4). In order to accomplish this, let $v \in W^{m, p}$ be given. Then it follows from (3.1), (2.4), and (1.2) that

$$
\begin{aligned}
& \sum_{1 \leq|\alpha| \leq|m|} \int_{\Omega} A_{\alpha}\left(x, \xi_{m}\left(u_{n_{k}}\right)\right) D^{\alpha} v+n_{k}^{-1} \int_{\Omega} \operatorname{sgn} u_{n_{k}}\left|u_{n_{k}}\right|^{p-1} v \\
& =G(v)+\int_{\Omega} f^{n_{k}}\left(x, u_{n_{k}}\right) v .
\end{aligned}
$$

From (3.20), we see that $\left\|u_{n_{k}}\right\|_{L^{p}} \leq K_{9}$ for $k=1,2, \ldots$. Hence it follows from Hölder's inequality and $v \in W^{m, p}$ that

$$
\lim _{k \rightarrow \infty}\left(n_{k}\right)^{-1} \int_{\Omega} \operatorname{sgn} u_{n_{k}}\left|u_{n_{k}}\right|^{p-1} v=0 .
$$

Next, from (f-2) we see that a.e. in $\Omega$

$$
\left|f^{n}\left(x, u_{n}\right)\right| \leq h_{1}(x)+K^{\prime}\left|u_{n}\right|^{q-1} \text { for } k=1,2, \ldots,
$$

where $h_{1} \in L^{q^{\prime}}$. Also, we see from Hölder's inequality that

$$
\int_{E}\left|u_{n}\right|^{q-1}|v| \leq\left\{\int_{\Omega}\left|u_{n}\right|^{q}\right\}^{(q-1) / q}\left\{\int_{E}|v|^{q}\right\}^{1 / q}
$$

where $E$ is a measurable subset of $\Omega$. From (2.3) and (3.20), we see that the first integral on the right-hand side of the inequality in (3.43) is uniformly bounded in $n$. Hence it follows from (3.42) and (3.43) that

$$
\left\{f^{n}\left(x, u_{n}\right) v\right\}_{n=1}^{\infty} \text { is absolutely equi-integrable. }
$$

Likewise, we see from (f-1), (2.2), and (3.25) that

$$
\lim _{n \rightarrow \infty} f^{n}\left(x, u_{n}\right) v(x)=f\left(x, u^{\#}\right) v(x) \text { a.e. in } \Omega \text {. }
$$

It consequently follows from Egoroff's theorem, (3.44), and (3.45) that

$$
\lim _{k \rightarrow \infty} \int_{\Omega} f^{n_{k}}\left(x, u_{n_{k}}\right) v=\int_{\Omega} f\left(x, u^{\#}\right) v .
$$

Next, with $\left\{u_{n_{k}}\right\}_{k=1}^{\infty}$ the subsequence given in (3.26), we obtain from (A-1), (3.25), and (3.26) that

$$
\begin{aligned}
& \lim _{k \rightarrow \infty} A_{\alpha}\left(x, \xi_{m}\left(u_{n_{k}}(x)\right)\right) D^{\alpha} v(x) \\
& \quad=A_{\alpha}\left(x, \xi_{m}\left(u^{\#}(x)\right)\right) D^{\alpha} v(x) \text { a.e. in } \Omega
\end{aligned}
$$

for $1 \leq|\alpha| \leq m$. Also, we see from (3.20) and (A-2) that (3.31) holds. Hence it follows from Hölder's inequality that $(2.17)^{\prime}$ holds with $K_{10}$ and $n_{k}$ replacing $K_{4}$ and $k$ respectively. Consequently

$$
\left\{A_{\alpha}\left(x, \xi_{m}\left(u_{n_{k}}\right)\right) D^{\alpha} v\right\}_{n=1}^{\infty} \text { is absolutely equi-integrable }
$$


for $1 \leq|\alpha| \leq m$. Hence it follows from (3.47), (3.48), and Egoroff's theorem that

$$
\lim _{k \rightarrow \infty} \int_{\Omega} A_{\alpha}\left(x, \xi_{m}\left(u_{n_{k}}\right)\right) D^{\alpha} v=\int_{\Omega} A_{\alpha}\left(x, \xi_{m}\left(u^{\#}\right)\right) D^{\alpha} v
$$

for $1 \leq|\alpha| \leq m$. From (3.40), (3.41), (3.46), and (3.49), we obtain that

$$
\sum_{1 \leq|\alpha| \leq m} \int_{\Omega} A_{\alpha}\left(x, \xi_{m}\left(u^{\#}\right)\right) D^{\alpha} v=G(v)+\int_{\Omega} f\left(x, u^{\#}\right) v \quad \forall v \in W^{m, p} .
$$

But from (1.2), we see that this last equality is the same as (1.4), and the proof of Theorem 1 is complete.

\section{Proof of Theorem 2}

To prove Theorem 2, for $n$ a positive integer, we set

$$
f_{n}(x, t)=f(x, t)-\operatorname{sgn} t|t|^{p-1} n^{-1} .
$$

It is clear $f_{n}(x, t)$ meets conditions $(\mathrm{f}-1)$. In (f-2) we have $q>p$ for $p \geq$ $N m^{-1}$ and $q=p N /(N-p m)$ for $1<p<N m^{-1}$. So $q>p$ also in this latter case. From the fact that $f(x, t)$ meets $(\mathrm{f}-2)$, it follows that

$$
\left|f_{n}(x, t)\right| \leq h_{1}(x)+K^{\prime}|t|^{q-1}+|t|^{p-1} n^{-1} \text { for a.e. } x \in \Omega \text { and } \forall t \in \mathbb{R} \text {, }
$$

where $h_{1} \in L^{q^{\prime}}$. Since $q-1>p-1$ in all cases, it follows from this last inequality that $f_{n}(x, t)$ also meets (f-2). Next, taking $\varepsilon=(2 n)^{-1}$ in (f-4), we see that

$$
t f_{n}(x, t) \leq h_{(2 n)^{-1}}^{*}(x)|t| \text { for a.e. } x \in \Omega \text { and }|t| \geq t_{0}(1 / 2 n) .
$$

Consequently, it follows from $(\mathrm{f}-2)$ that $f_{n}(x, t)$ also meets $(\mathrm{f}-3)$. We record all this as

$$
f_{n}(x, t) \text { meets conditions }(\mathrm{f}-1)-(\mathrm{f}-3) .
$$

Taking $\varepsilon=(2 n)^{-1}$ in (f-4) once again, we see from (4.1) that

$$
f_{n}(x, t) \leq-t^{p-1} / 2 n+h_{(2 n)^{-1}}^{*}(x)
$$

for $t \geq t_{0}(1 / 2 n)$ and a.e. $x \in \Omega$. It therefore follows that

$$
\limsup _{t \rightarrow \infty} f_{n}(x, t)=-\infty \text { for a.e. } x \in \Omega \text {. }
$$

In a similar manner, using $(\mathrm{f}-4)$ for $t \leq-t_{0}(1 / 2 n)$, we obtain that

$$
\liminf _{t \rightarrow-\infty} f_{n}(x, t)=\infty \text { for a.e. } x \in \Omega \text {. }
$$

Next, we observe that $G(1)$ is always finite-valued. It consequently follows from (4.1)-(4.4) and Theorem 1 that $\exists\left\{u_{n}\right\}_{n=1}^{\infty}$ with $u_{n} \in W^{m, p}$ s.t.

$$
\begin{aligned}
\mathscr{Q}\left(u_{n}, v\right)= & G(v)-n^{-1} \int_{\Omega} \operatorname{sgn} u_{n}\left|u_{n}\right|^{p-1} v \\
& +\int_{\Omega} f\left(x, u_{n}\right) v \quad \forall v \in W^{m, p} .
\end{aligned}
$$


We claim that

$$
\left\{\left\|u_{n}\right\|_{W^{m, p}}\right\}_{n=1}^{\infty} \text { is a uniformly bounded sequence. }
$$

Suppose the claim in (4.6) is false. Then without loss in generality, we can assume that

$$
\lim _{n \rightarrow \infty}\left\|u_{n}\right\|_{W^{m, p}}=\infty \text {. }
$$

Replacing $v$ by $u_{n}$ in (4.5) and using (A-4), we see that

$$
c_{0} \int_{\Omega}\left\{\sum_{1 \leq|\alpha| \leq m}\left|D^{\alpha} u_{n}\right|^{2}\right\}^{p / 2} \leq G\left(u_{n}\right)+\int_{\Omega} f\left(x, u_{n}\right) u_{n}+\int_{\Omega} Z(x) .
$$

Also, we note $\exists K_{12}$ such that

$$
|G(w)| \leq K_{12}\|w\|_{W^{m, p}} \quad \forall w \in W^{m, p} .
$$

We set

$$
v_{n}=u_{n} /\left\|u_{n}\right\|_{W^{m, p}} \text { for } n=1,2, \ldots
$$

and obtain from the last stated inequality on dividing both sides of (4.8) by $\left\|u_{n}\right\|_{W^{m, p}}^{p}$ that

$$
c_{0}\left\|D^{\alpha} v_{n}\right\|_{L^{p}}^{p} \leq\left\|u_{n}\right\|_{W^{m, p}}^{-p}\left[K_{12}\left\|u_{n}\right\|_{W^{m, p}}+\int_{\Omega} f\left(x, u_{n}\right) u_{n}+\int_{\Omega} Z(x)\right]
$$

for $1 \leq|\alpha| \leq m$.

To handle the first integral on the right-hand side of the inequality in (4.10), we proceed as follows. Given $\varepsilon>0$, we set

$$
B_{n, \varepsilon}=\left\{x:\left|u_{n}(x)\right| \leq t_{0}(\varepsilon)\right\},
$$

where $t_{0}(\varepsilon)$ is the constant in $(\mathrm{f}-4)$. We then have from (f-4) that

$$
\int_{\Omega} f\left(x, u_{n}\right) u_{n} \leq \int_{B_{n, \varepsilon}} f\left(x, u_{n}\right) u_{n}+\varepsilon \int_{\Omega}\left|u_{n}\right|^{p}+\int_{\Omega} h^{*}(x)\left|u_{n}\right| .
$$

It consequently follows from (4.11), (f-2), Hölder's inequality, (2.3), and (4.7) all applied to this last inequality that

$$
\limsup _{n \rightarrow \infty}\left\|u_{n}\right\|_{W^{m, p}}^{-p} \int_{\Omega} f\left(x, u_{n}\right) u_{n} \leq \varepsilon
$$

Since $\varepsilon$ is an arbitrary positive number, we conclude

$$
\underset{n \rightarrow \infty}{\limsup }\left\|u_{n}\right\|_{W^{m, p}}^{-p} \int_{\Omega} f\left(x, u_{n}\right) u_{n} \leq 0
$$

Recalling that $c_{0}$ is a positive constant and that $Z \in L^{1}$, we see from (4.10) and (4.12) that

$$
\lim _{n \rightarrow \infty}\left\|D^{\alpha} v_{n}\right\|_{L^{p}}=0 \quad \text { for } 1 \leq|\alpha| \leq m
$$


Now from (2.1) and (4.9) we see that

$$
1=\left\|v_{n}\right\|_{W^{m, p}}^{p}=\left\|v_{n}\right\|_{L^{p}}^{p}+\sum_{1 \leq|\alpha| \leq m}\left\|D^{\alpha} v_{n}\right\|_{L^{p}}^{p} .
$$

Hence, from (4.13) we obtain

$$
\lim _{n \rightarrow \infty}\left\|v_{n}\right\|_{L^{p}}=1 \text {. }
$$

Next, as in the proof of Theorem 1, we use the compact imbedding theorem [ 1 , p. 144] along with the fact that $W^{m, p}$ is a separable reflexive Banach space to obtain (3.9)-(3.14). In particular from (3.10), we have that

$$
\lim _{n \rightarrow \infty}\left\|D^{\alpha} v_{n}-D^{\alpha} v_{0}\right\|_{L^{p}}=0
$$

for $|\alpha|=1$. Hence we conclude from (4.13) that $D^{\alpha} v_{0}=0$ a.e. in $\Omega$ for $|\alpha|=1$. Since $\Omega$ is a bounded open connected set, it follows that $v_{0}=$ constant a.e. in $\Omega$. From (3.10) and (4.13)-(4.15) we see that $\left\|v_{0}\right\|_{L^{p}}=1$. Therefore $v_{0}=[\text { meas } \Omega]^{-1 / p}$ or $v_{0}=-[\text { meas } \Omega]^{-1 / p}$ a.e. in $\Omega$. We shall suppose the former case holds and conclude the proof to the theorem on this basis. In case the latter case holds, a similar proof prevails. Therefore we have that

$$
v_{0}=[\text { meas } \Omega]^{-1 / p} \text { a.e. in } \Omega \text {. }
$$

Also from (3.13) we see that

$$
\lim _{n \rightarrow \infty} v_{n}(x)=[\text { meas } \Omega]^{-1 / p} \quad \text { a.e. in } \Omega \text {. }
$$

Next taking $v=u_{n}$ once again in (4.5), we see from (A-4) that

$$
0 \leq G\left(u_{n}\right)+\int_{\Omega} f\left(x, u_{n}\right) u_{n}+\int_{\Omega} Z(x) \text {. }
$$

Also, taking $\varepsilon=1$ in both (f-2) and (f-4), we see that $\exists h^{* *} \in L^{q^{\prime}}$ s.t.

$$
t f(x, t) \leq|t|^{p}+h^{* *}(x)|t| \quad \forall t \in \mathbb{R} \text { and a.e. } x \in \Omega \text {. }
$$

Using (4.19) in conjunction with (4.18), we obtain

$$
\begin{aligned}
& \int_{\Omega}\left\{\left|u_{n}\right|^{p}+h^{* *}(x)\left|u_{n}\right|-f\left(x, u_{n}\right) u_{n}\right\} \\
& \leq G\left(u_{n}\right)+\int_{\Omega}\left[\left|u_{n}\right|^{p}+h^{* *}(x) u_{n}\right]+\int_{\Omega} Z(x),
\end{aligned}
$$

where all integrands are nonnegative a.e. in $\Omega$.

Now, from (2.3), (4.7), and Hölder's inequality, we see that

$$
\lim _{n \rightarrow \infty}\left\|u_{n}\right\|_{W^{m, p}}^{-p} \int_{\Omega} h^{* *}(x)\left|u_{n}\right|=0
$$

Likewise, we see from $(4.8)^{\prime}$ and (4.7) that

$$
\lim _{n \rightarrow \infty} G\left(u_{n}\right)\left\|u_{n}\right\|_{W^{m, p}}^{-p}=0
$$


Hence dividing both sides of (4.20) by $\left\|u_{n}\right\|_{W^{m, p}}^{p}$ and using (4.7), (4.15), (4.21), and (4.22), we obtain

$$
\liminf _{n \rightarrow \infty} \int_{\Omega}\left\|u_{n}\right\|_{W^{m, p}}^{-p}\left\{\left|u_{n}\right|^{p}+h^{* *}(x)\left|u_{n}\right|-f\left(x, u_{n}\right) u_{n}\right\} \leq 1 .
$$

From (4.19) we see, for every $n$, the integrand in this last inequality is nonnegative a.e. in $\Omega$. Hence, it follows from Fatou's lemma [11, p. 24] that

$$
\int_{\Omega} \liminf _{n \rightarrow \infty}\left\|u_{n}\right\|_{W^{m, p}}^{-p}\left\{\left|u_{n}\right|^{p}+h^{* *}(x)\left|u_{n}\right|-f\left(x, u_{n}\right) u_{n}\right\} \leq 1 .
$$

From (4.17) we therefore have that

$$
\int_{\Omega}\left\{[\text { meas } \Omega]^{-1}-\limsup _{n \rightarrow \infty} f\left(x, u_{n}\right) u_{n}\left\|u_{n}\right\|_{W^{m, p}}^{-p}\right\} \leq 1 .
$$

Now $u_{n}(x)=\left\|u_{n}\right\|_{W^{m, p}} v_{n}(x)$. Consequently it follows from (4.7) and (4.17) that $\lim _{n \rightarrow \infty} u_{n}(x)=\infty$ a.e. in $\Omega$. Also,

$$
f\left(x, u_{n}\right) u_{n}(x)\left\|u_{n}\right\|_{W^{m, p}}^{-p}=f\left(x, u_{n}\right)\left|v_{n}(x)\right|^{p} / \operatorname{sgn} u_{n}(x)\left|u_{n}(x)\right|^{p-1} .
$$

We consequently obtain from (1.10), (4.17), and (4.23) that

$$
\int_{\Omega}\left\{[\text { meas } \Omega]^{-1}-[\text { meas } \Omega]^{-1} \mathscr{F}_{+}(x)\right\} \leq 1 .
$$

But then $\int_{\Omega} \mathscr{F}_{+}(x) \geq 0$ and this is a direct contradiction of the first inequality in assumption (1.11) in the hypothesis of Theorem 2. We conclude that (4.7) is false and the claim in (4.6) is true.

The claim in (4.6) in the proof of this theorem corresponds to the claim in (3.2) and (3.20) in the proof of Theorem 1. A close reading of the proof of Theorem 1 shows that from (3.20) forward except for (3.39)(ii), the proof only makes use of conditions ( $\mathrm{f}-1)$ and $(\mathrm{f}-2)$ when dealing with $f^{n}\left(x, u_{n}\right)$. The analogue of (3.39)(ii) for the proof of Theorem 2 is: given $\varepsilon>0, \exists \delta>0$ such that if (meas $E)<\delta$ then $\int_{E} f\left(x, u_{n}\right) u_{n}<\varepsilon$ for $n=1,2, \ldots$. But this fact follows easily from (f-4), (4.6), (2.3), and Hölder's inequality. Since (f-1) and $(\mathrm{f}-2)$ are part of the conditions in the hypothesis of Theorem 2, we see therefore that the completion of the proof of Theorem 2 is the same as that for Theorem 1 from (3.20) forward. Hence the proof of Theorem 2 is complete.

\section{A CONCLUDING REMARK}

In this section, we prove a remark which shows that under certain special circumstances, condition (1.3) in the statement of Theorem 1 can be both necessary and sufficient for the solution of (1.4).

Remark. Let $1<p<\infty$ and let $\Omega$ be a bounded open connected set with the cone property. Suppose that $\mathscr{Q}(u, v)$ is given by (1.2) where $A_{\alpha}\left(x, \xi_{m}\right)$ satisfies (A-1)-(A-4) for $1 \leq|\alpha| \leq m$ and that $f(t)$ is a bounded continuous function defined on $\mathbb{R}$. Suppose also that $\lim _{t \rightarrow \pm \infty} f(t)=f( \pm)$ exists and that 
$f(+)<f(t)<f(-)$ for $t \in \mathbb{R}$. Then a necessary and sufficient condition that $\exists u^{\#} \in W^{m, p}$ such that

$$
\mathscr{Q}\left(u^{\#}, v\right)=G(v)+\int_{\Omega} f\left(u^{\#}\right) v \quad \forall v \in W^{m, p}
$$

is that

$$
f(+)<-[\text { meas } \Omega]^{-1} G(1)<f(-) .
$$

That condition (5.2) is sufficient follows immediately from Theorem 1 because the conditions in the hypothesis of the remark imply those of Theorem 1 and (5.2) in this case is the same as (1.3).

To see that condition (5.2) is necessary, suppose $u^{\#} \in W^{m, p}$ exists satisfying (5.1). Setting $v=1$ in (5.1), we see from (1.2) that

$$
-G(1)=\int_{\Omega} f\left(u^{\#}\right)
$$

By hypothesis $f(+)<f\left(u^{\#}(x)\right)<f(-)$ for a.e. $x \in \Omega$. Hence $\int_{\Omega} f(+)<$ $\int_{\Omega} f\left(u^{\#}\right)<\int_{\Omega} f(-)$. This fact combined with (5.3) gives (5.2) and the proof of the remark is complete.

\section{REFERENCES}

1. R. A. Adams, Sobolev spaces, Academic Press, New York, 1975.

2. S. Agmon, Lectures on elliptic boundary value problems, Van Nostrand, Princeton, N. J., 1965.

3. F. E. Browder, Existence theorems in partial differential equations, Proc. Sympos. Pure Math., vol. 16, Amer. Math. Soc., Providence, R. I., 1970, pp. 1-60.

4. D. G. de Figueiredo and J. P. Gossez, Nonlinear perturbations of a linear elliptic problem near its first eigenvalue, J. Differential Equations 30 (1978), 1-19.

5. B. Epstein, Partial differential equations, McGraw-Hill, New York, 1962.

6. D. Gilbarg and N. S. Trudinger, Elliptic partial differential equations of second order, 2nd ed., Springer-Verlag, Berlin, 1983.

7. O. A. Ladyzhenskaya and N. N. Uraltseva, Linear and quasilinear elliptic equations, Academic Press, New York, 1968.

8. E. M. Landesman and A. C. Lazer, Nonlinear perturbations of linear elliptic boundary value problems at resonance, J. Math. Mech. 19 (1970), 609-623.

9. C. B. Morrey, Multiple integrals in the calculus of variations, Springer-Verlag, Berlin, 1966.

10. J. Necas, Introduction to the theory of nonlinear elliptic equations, Wiley-Interscience, New York, 1986.

11. W. Rudin, Real and complex analysis, 2nd ed., McGraw-Hill, New York, 1974.

Department of Mathematics, University of California, Riverside, California 92521 